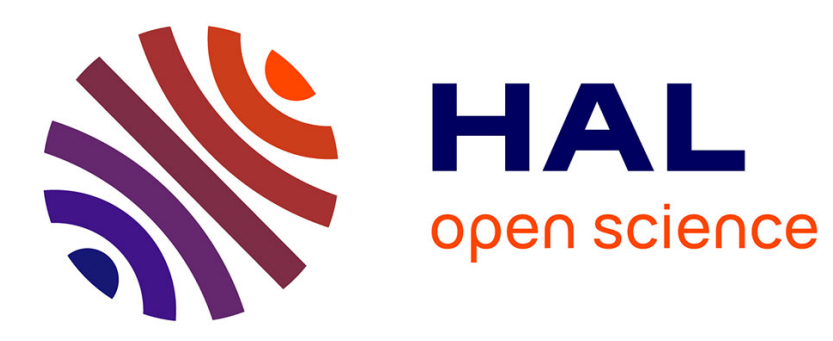

\title{
Utilisation d'axes embryonnaires de pêcher pour apprécier l'effet d'un milieu nutritif minéral sur le développement des tiges ou des racines en culture in vitro
}

Antonio Tombolato, René Monet

\section{To cite this version:}

Antonio Tombolato, René Monet. Utilisation d'axes embryonnaires de pêcher pour apprécier l'effet d'un milieu nutritif minéral sur le développement des tiges ou des racines en culture in vitro. Agronomie, 1984, 4 (10), pp.927-931. hal-00884595

\section{HAL Id: hal-00884595 \\ https://hal.science/hal-00884595}

Submitted on 1 Jan 1984

HAL is a multi-disciplinary open access archive for the deposit and dissemination of scientific research documents, whether they are published or not. The documents may come from teaching and research institutions in France or abroad, or from public or private research centers.
L'archive ouverte pluridisciplinaire HAL, est destinée au dépôt et à la diffusion de documents scientifiques de niveau recherche, publiés ou non, émanant des établissements d'enseignement et de recherche français ou étrangers, des laboratoires publics ou privés. 


\section{Utilisation d'axes embryonnaires de pêcher pour apprécier l'effet d'un milieu nutritif minéral sur le développement des tiges ou des racines en cul- ture in vitro}

Antonio TOMBOLATO \& René MONET $\left(^{*}\right)$

Institut Agronomique, Section Fruits des climats tempérés, 13100 Campinas S.P. (Brésil)

(*) I.N.R.A., Station d'Arboriculture fruitière, Centre de Recherches de Bordeaux, F 33140 Pont-de-la-Maye

RÉSUMÉ

Les axes embryonnaires des graines mûres de pêcher peuvent être utilisés pour révéler l'aptitude d'un milieu nutritif, sans substances de croissance, à favoriser le développement préférentiel des racines ou des tiges en culture in vitro. De ce fait, ils peuvent aussi être utilisés pour la mise au point de milieux nutritifs destinés à la culture in vitro d'embryons.

Mots clés additionnels : Culture d'embryons, sélection du pêcher. development of stems or roots in in vitro culture.

The embryonic axes (embryos with cotyledons removed) from ripe peach seeds can be used to test the suitability of a nutrient medium, without growth substances, for favouring the preferential development of roots or stems in in vitro culture. They can accordingly be used to improve nutrient media for embryo culture.

Additional key words : Embryo culture, peach breeding.

\section{INTRODUCTION}

Chez le pêcher, la période la plus active du développement de l'embryon se situe peu après le durcissement du noyau. Cette phase, qui dure plus d'un mois chez les variétés de saison, est réduite à quelques jours chez les variétés à maturité précoce, ce qui provoque l'avortement de l'embryon et l'impossibilité d'obtenir en conditions naturelles des descendants à partir de géniteurs femelles très précoces.

Les sélectionneurs ont eu, très tôt, recours aux cultures in vitro des embryons immatures de pêcher pour pallier cet inconvénient (DAVIDSON, 1933; LAMMERTS, 1942 ; LESLEY \& BONNER, 1952 ; THEOBALD \& Hough, 1960 ; MoneT, 1968). Cependant, par cette technique, il est impossible d'obtenir une survie d'embryons dont la taille soit inférieure à $5 \mathrm{~mm}$ environ à moins d'avoir recours, comme l'ont proposé TOLEDO et al. (1980), à une culture in vitro préalable de la graine. Ce procédé est malheureusement peu pratique et ne peut s'appliquer qu'à un nombre limité de graines : il faut désinfecter les fruits, en extraire les graines et les cultiver sur milieu nutritif approprié, enfin extraire l'embryon de la graine et le repiquer sur un nouveau milieu pour obtenir sa germination.

La taille de $5 \mathrm{~mm}$ est approximativement atteinte à la fin de l'organogenèse embryonnaire ; au-delà de ce seuil, l'embryon accumule les réserves, les cotylédons vont grossir et occupent peu à peu la place du nucelle et de l'albumen (graine exalbuminée). C'est au cours de cette période que la survie de l'embryon devient possible en culture in vitro, mais elle dépend beaucoup de la taille des cotylédons et des conditions de culture.

Le comportement des embryons du pêcher est donc nettement n.oins bon, en culture in vitro, que celui d'autres espèces, telles que le cotonnier (MAUNEY, 1961) ou la capselle (MONNIER, 1976), chez lesquelles la survie a pu être obtenue avec des embryons en cours d'organogenèse. 
Pour le sélectionneur, il n'en demeure pas moins important d'obtenir un taux élevé de survie d'embryons mis en culture à la fin de l'organogenèse embryonnaire car, chez la plupart des géniteurs précoces utilisés, les embryons ont atteint ou dépassé ce stade lorsque le fruit est mûr sur l'arbre.

Il n'y a pas eu de recherche systématique d'un milieu nutritif permettant d'assurer le taux maximum de survie de ces embryons ; chaque laboratoire a son milieu préféré sans que l'on sache quel est le meilleur.

Pour comparer des milieux nutritifs, on se heurte d'ailleurs à deux difficultés : on ne peut disposer des embryons que sur une très courte période de l'année et leur état de développement est très hétérogène. Pour éviter ces difficultés, nous avons pensé remplacer ces embryons immatures par des axes embryonnaires (embryons privés de leurs cotylédons) prélevés sur des graines mûres, génétiquement proches de l'homozygotie. L'axe embryonnaire associant radicule et gemmule va extérioriser les effets du milieu nutritif sur ces deux parties essentielles d'une plantule; par ailleurs, la suppression des cotylédons rapproche l'axe embryonnaire de l'état physiologique d'un embryon immature au moins pour ce qui est de sa phase postorganogène, lorsqu'il accumule des réserves. Nous présentons ci-dessous quelques résultats obtenus avec ce matériel.

\section{MATÉRIEL ET MÉTHODES}

Plusieurs solutions minérales ont été comparées, soit en milieu solidifié par la gélose, soit en milieu liquide agité. Leur composition ionique exprimée en meq/l est donnée dans le tableau 1.

Pour les cultures en milieu solide, nous utilisons des flacons de prélèvements dits de kola d'une contenance de $100 \mathrm{ml}$. Ces flacons sont fermés hermétiquement par une capsule d'aluminium munie d'un joint de caoutchouc. Chaque flacon contient $20 \mathrm{ml}$ de milieu.

Pour les cultures en milieu liquide, nous utilisons des piluliers d'une capacité de $20 \mathrm{ml}$ obturés par une capsule de plastique permettant les échanges gazeux.

Chaque pilulier reçoit $3 \mathrm{ml}$ de milieu ; il est fixé sur un agitateur dont la rotation dans le plan se fait à la vitesse de 90 tours $/ \mathrm{mn}$.

La source de carbone est le saccharose utilisé à 3 p. 100 , le pH du milieu est ajusté à 5,8 par une solution décinormale de soude ou d'acide chlorhydrique.

Les milieux de culture sont stérilisés dans leurs récipients à $110^{\circ} \mathrm{C}$ pendant $30 \mathrm{mn}$. Seules les capsules de plastique, ne résistant pas à ces températures, sont sté- rilisées par immersion dans l'alcool à 95 p. 100 pendant $24 \mathrm{~h}$.

Les manipulations aseptiques concernant la mise en culture elle-même sont réalisées sous hotte à flux laminaire.

Les axes embryonnaires (fig. 1) sont prélevés sur des graines récoltées l'année précédente sur le pêcher porte-greffe «GF 305 » qui est proche d'une lignée pure. Ces graines ont été conservées dans leur noyau dans un local sec et frais $\left(15^{\circ} \mathrm{C}\right.$ environ) après leur récolte.

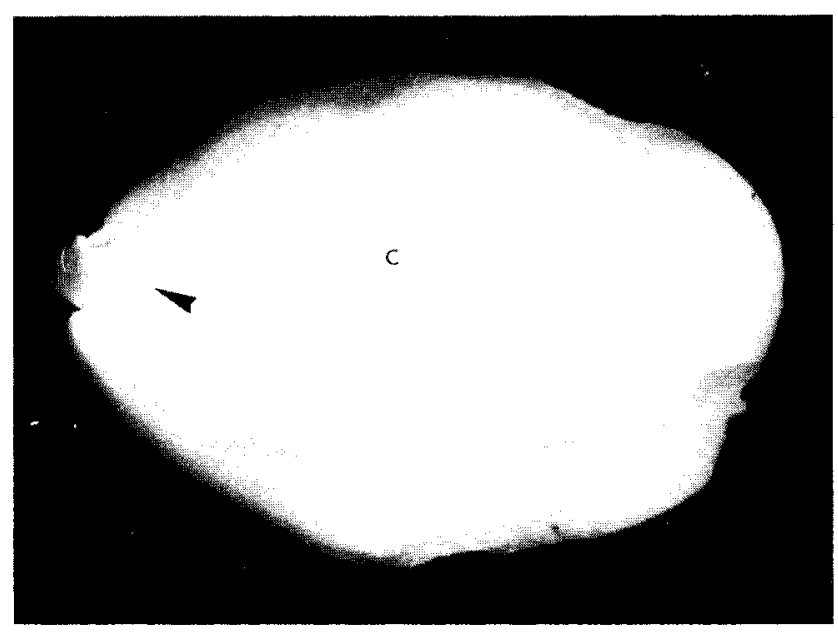

Figure 1

Graine de pêcher privée des téguments et d'un cotylédon $(\times 4)$. Axe embryonnaire (flèche), cotylédons (C).

Peach seed without teguments and one cotyledon removed $(\times 4)$. Embryonic axis (arrow), cotyledon (C).

La veille de la mise en culture, les noyaux sont cassés ; les graines sont désinfectées pendant $1 \mathrm{~h}$ dans une solution d'hypochlorite de calcium à 8 p. 100 dans laquelle on ajoute $0,5 \mathrm{ml}$ de mouillant (TWEEN 20). Ces graines sont ensuite rincées dans l'eau stérile et maintenues jusqu'au lendemain pour imbibition dans une boîte de Petri garnie d'eau.

Le prélèvement des axes se fait en ouvrant la graine à l'aide de pinces chirurgicales et en détachant l'axe embryonnaire du cotylédon auquel il adhère encore, à l'aide d'un scalpel. Les axes, d'abord stockés dans de l'eau stérile, sont ensuite placés sur différents milieux de culture. Au moment du prélèvement, leur poids moyen frais est de $1,5 \mathrm{mg}$, leur poids moyen sec de $0,58 \mathrm{mg}$ et leur longueur moyenne est de $2,3 \mathrm{~mm}$.

La culture se poursuit pendant 19 ou $20 \mathrm{j}$ en salle climatisée à $25^{\circ} \mathrm{C}$ et éclairage continu de 3000 lux.

TABLEAU 1

Composition ionique des milieux de culture (meq/l).

Ionic composition of nutrient media (meq/l).

\begin{tabular}{|c|c|c|c|c|c|c|c|c|c|}
\hline & $\mathrm{NO}_{3}^{-}$ & $\mathrm{PO}_{4} \mathrm{H}_{2}^{-}$ & $\mathrm{SO}_{4}^{-}$ & $\mathrm{Cl}^{-}$ & $\mathrm{K}^{+}$ & $\mathrm{Ca}^{++}$ & $\mathrm{Na}^{+}$ & $\mathrm{Mg}^{++}$ & $\mathrm{NH}_{4}^{-}$ \\
\hline KNOP $1 / 2$ & 5,5 & 0,9 & 1,0 & 0 & 2,2 & 4,2 & 0 & 1,0 & 0 \\
\hline WHITE & 3,0 & 0,1 & 8,7 & 0,9 & 1,7 & 1,9 & 2,85 & 5,85 & 0 \\
\hline Murashige-SKOOG & 38,6 & 1,25 & 3,5 & 5,9 & 20,1 & 5,9 & 0 & 3,0 & 20,6 \\
\hline SCHENK-HILDEBRANDT & 24,75 & 2,6 & 3,5 & 2,7 & 24,8 & 2,7 & 0,1 & 3,25 & 2,6 \\
\hline Nitsch-Nitsch & 18,4 & 0,5 & 2,0 & 1,1 & 9,9 & 2,2 & 0 & 1,5 & 9 \\
\hline Milleer & 29,5 & 22 & 1,2 & 0,9 & 13,0 & 3,3 & 0 & 0,6 & 12,5 \\
\hline
\end{tabular}


On procède ensuite à l'observation des axes et éventuellement à leur repiquage en serre.

Nous avons, pour les 2 premiers essais, établi la moyenne arithmétique des poids frais des plantules et, pour un $3^{e}$ essai, celle des poids secs des tiges et des racines séparément. Un jugement visuel du comportement des plantules dans les différents milieux a été fait.

\section{RÉSULTATS ET DISCUSSION}

\section{A. Effet quantitatif des milieux}

Les solutions minérales utilisées en culture d'embryons de pêcher sont très variées ; on peut distinguer les solutions faiblement concentrées et les solutions fortement concentrées. D'autre part, la culture sur milieu liquide agité est rarement utilisée. Nous avons donc comparé, dans un $1^{\text {er }}$ essai, 5 solutions minérales (macro- et microéléments) en milieu liquide et en milieu gélosé à $0,8 \mathrm{p} .100$ d'agar. Deux de ces solutions ont une concentration ionique faible :

- KNOP 1/2 - microéléments de HELLER (1953),

- White (1936).

Les 3 autres solutions ont une concentration ionique forte :

- MURASHIGE-SKOOG (1962),

- SCHENK-HILDEBRANDT (1972),

- NiTSCH-NITSCH (1969).

Les résultats obtenus après $19 \mathrm{j}$ de culture sont rassemblés dans le tableau 2.

\section{TABLEAU 2}

Poids frais moyen, en mg, des axes embryonnaires cultivés dans différentes solutions minérales sur milieu gélosé à 0,8 p. 100 d'agar et milieu liquide après $19 j$ de culture.

Average fresh weight in $\mathrm{mg}$ of embryonic axes cultured on several mineral solutions on agar medium $(0.8 \%)$ and on liquid medium after 19 days of culture.

\begin{tabular}{lcc}
\hline \multicolumn{1}{c}{ Solutions minérales } & $\begin{array}{c}\text { Nombre d'axes } \\
\text { embryonnaires }\end{array}$ & $\begin{array}{c}\text { Poids frais } \\
\text { moyen (mg) }\end{array}$ \\
\hline Milieu gélosé (0,8 p. 100) & & \\
KNOP 1/2-HELLER & 14 & 3,6 \\
WHITE & 18 & 3,9 \\
MURASHIGE-SKOOG & 15 & 6,3 \\
SCHENK-HILDEBRANDT & 14 & 7,4 \\
NiTSCH-NITSCH & 15 & 7,0 \\
& & \\
Milieu liquide & & \\
KNOP 1/2-HELLER & 9 & 12,9 \\
WHITE & 9 & 12,1 \\
MURASHIGE-SKOOG & 9 & 19,9 \\
SCHENK-HILDEBRANDT & 10 & 22,0 \\
NITSCH-NITSCH & 9 & 19,0 \\
\hline \hline
\end{tabular}

On constate les faits suivants :

- Les poids frais des axes embryonnaires cultivés en milieu liquide (quelle que soit la formule utilisée) sont en moyenne 3 fois supérieurs à ceux des axes embryonnaires cultivés sur milieu gélosé.

- Les milieux se partagent en 2 groupes d'efficacité comparable : les milieux qui permettent une synthèse importante de matière fraîche par unité de temps (MURASHIGE-SKOOG, SCHENK-HILDEBRANDT, NITSCH-NITSCH) ; les milieux nettement moins favorables à cette synthèse (KNOP 1/2-HELLER, WHITE).

Si l'on pouvait prévoir la meilleure efficacité des milieux « riches » par rapport aux milieux «pauvres » (les milieux de KNOP $1 / 2$ et de WHITE sont pratiquement abandonnés en culture de tissus), la supériorité des milieux liquides agités sur les milieux gélosés est assez inattendue. N'y a-t-il pas une rétention des solutions par le gel peut-être trop concentré en agar ? Nous avons voulu préciser ce point par un nouvel essai dans lequel nous avons conservé les 3 milieux " riches » précédemment utilisés (MURASHIGE-SKOOG, SCHENK-HILDEBRANDT, NITSCH-NITSCH) auxquels nous avons adjoint, à titre comparatif, 2 nouveaux milieux particulièrement riches, ceux de MILLER (1965) et de QUOIRIN-LEPOIVRE (1977).

Dans les milieux solides, la teneur en gélose a été réduite de moitié passant ainsi à 0,4 p. 100 d'agar. Le tableau 3 rassemble les résultats de l'essai après $20 \mathrm{j}$ de culture. On constate que :

- la diminution de la concentration en agar est favorable dans toutes les formules essayées. L'écart entre le poids frais moyen des axes cultivés sur milieu gélosé et sur milieu liquide agité s'est amoindri sans toutefois qu'il disparaisse.

- L'efficacité des 3 premières formules MURASHIGE-SKOOG, SCHENK-HILDEBRANDT, NITSCHNitSCH est comparable à celle du $1^{\text {er }}$ essai.

En ce qui concerne les 2 formules nouvelles, celle de MILlER induit un développement très irrégulier (un axe était anormalement développé, les autres, au contraire, n'avaient qu'un très faible développement), celle de QUOIRIN-LEPOIVRE permet une production importante de matière fraîche mais les axes embryonnaires sont chlorotiques.

Il apparaît, avec les deux derniers milieux notamment, une notion d'effet qualitatif que nous allons examiner maintenant.

TABLEAU 3

Poids frais moyen, en $m g$, des axes embryonnaires cultivés dans diverses solutions minérales sur milieu gélosé à $0,4 \mathrm{p}$. $100 \mathrm{~d}$ 'agar et milieu liquide après $20 j$ de culture.

Average fresh weight in mg of embryonic axes cultured on several mineral solutions on agar medium $(0.4 \%)$ and on liquid medium after 20 days of culture.

\begin{tabular}{lcc}
\hline \hline \multicolumn{1}{c}{ Solutions minérales } & $\begin{array}{c}\text { Nombre d'axes } \\
\text { embryonnaires }\end{array}$ & $\begin{array}{c}\text { Poids frais } \\
\text { moyen (mg) }\end{array}$ \\
\hline Milieu gélosé (0,4 p. 100) & & \\
MuRASHIGE-SKOOG & 12 & 22,4 \\
SCHENK-HILDEBRANDT & 12 & 18,7 \\
NITSCH-NITSCH & 11 & 17,4 \\
MILLER & 11 & 17,6 \\
QUOIRIN-LEPOIVRE & 12 & 21,6 \\
& & \\
Milieu liquide & & \\
MURASHIGE-SKOOG & 12 & 26,9 \\
SCHENK-HILDEBRANDT & 12 & 20,5 \\
NITSCH-NITSCH & 8 & 22,4 \\
MILLER & 5 & 37,0 \\
QUOIRIN-LEPOIVRE & 8 & 22,8 \\
\hline \hline
\end{tabular}




\section{B. Effet qualitatif des milieux}

Le choix d'un milieu ne peut se faire uniquement sur la masse de matière fraîche qu'il permet d'obtenir par unité de temps. Il importe en effet de savoir si le milieu induit un développement équilibré de la partie aérienne et de la partie racinaire de l'axe embryonnaire ou si, au contraire, il est plus favorable au développement de la racine ou de la tige. Par ailleurs, un milieu peut provoquer des anomalies nutritionnelles (déformations, chloroses, etc...) qu'il est souhaitable de connaître. C'est ce qui nous a conduit à réaliser un $3^{\mathrm{e}}$ essai dans lequel cet aspect a été spécialement étudié.

Dans cet essai, nous avons utilisé les 3 formules les plus performantes des essais précédents, MURASHIGESKOOG, SCHENK- HILDEBRANDT, NITSCH-NITSCH et, pour avoir une opinion définitive, nous avons repris la solution de MILLER. Ces formules ont été utilisées en milieu liquide agité et en milieu gélosé à 0,4 et 0,6 p. 100 d'agar. Après $20 \mathrm{j}$ de culture, nous avons établi les poids moyens de la matière sèche des racines et des tiges de chaque échantillon et noté l'aspect des plantules.

Le tableau 4 rassemble les résultats de cet essai. On constate que le classement des formules en ce qui concerne les rapports entre le poids de matière sèche des racines et des tiges est sensiblement le même sur milieu liquide que sur milieu solide. On retrouve encore l'effet défavorable d'une concentration trop forte en agar : le poids total des axes est toujours plus faible sur le milieu dont la concentration en agar est la plus forte. Enfin le rapport racine/tige s'accroît avec l'enrichissement du milieu en agar comme si la difficulté d'extraction des substances nutritives du milieu avait pour effet de favoriser le développement du système absorbant.

\section{TABLEAU 4}

Poids sec moyen, en $m g$, des tiges et des racines des axes embryonnaires cultivés dans diverses solutions minérales en milieu gélosé à $0,4 \mathrm{p} .100$ et à $0,6 \mathrm{p}$. $100 \mathrm{~d}$ 'agar et en milieu liquide après $20 \mathrm{j}$ de culture.

Average dry weight in $\mathrm{mg}$ of stems and roots of embryonic axes cultured on several mineral solutions on agar media $(0.4 \%, 0.6 \%)$ and in liquid medium after 20 days of culture.

\begin{tabular}{|c|c|c|c|c|}
\hline \multirow{2}{*}{ Solutions minérales } & \multirow{2}{*}{$\begin{array}{c}\text { Nombre } \\
\text { d'axes } \\
\text { embryon. }\end{array}$} & \multicolumn{2}{|c|}{ Poids sec moyen (mg) } & \multirow{2}{*}{$\begin{array}{l}\text { Rapport } \\
\text { racine/ } \\
\text { tige }\end{array}$} \\
\hline & & racines & tiges & \\
\hline \multicolumn{5}{|l|}{ Milieu gélosé $(0,4$ p. 100) } \\
\hline Murashige-SkoGG & 12 & 1,41 & 0,77 & 1,83 \\
\hline SCHENK-HILDEBRANDT & 13 & 1,39 & 1,03 & 1,34 \\
\hline NitSCH-NiTSCH & 9 & 1,40 & 0,58 & 2,41 \\
\hline Miller & 11 & 0,99 & 0,70 & 1,41 \\
\hline \multicolumn{5}{|l|}{ Milieu gélosé $(0,6$ p. 100) } \\
\hline Murashige-SKOGG & 9 & 1,32 & 0,58 & 2,28 \\
\hline SCHENK-HILDEBRANDT & 10 & 0,98 & 0,73 & 1,34 \\
\hline Nitsch-Nitsch & 12 & 1,12 & 0,47 & 2,38 \\
\hline MiLleR & 12 & 0,98 & 0,49 & 2,00 \\
\hline \multicolumn{5}{|l|}{ Milieu liquide } \\
\hline MuRAshige-SKOOG & 11 & 2,30 & 1,66 & 1,38 \\
\hline SCHENK-HILDEBRANDT & 9 & 2,44 & 2,90 & 0,84 \\
\hline Nitsch-Nitsch & 10 & 3,86 & 1,94 & 1,99 \\
\hline Miller & 8 & 1,93 & 1,11 & 1,74 \\
\hline
\end{tabular}

On peut aussi caractériser chaque milieu en fonction de son aptitude à favoriser le développement de la racine ou de la tige et en fonction de l'aspect des axes embryonnaires à la fin de l'essai :

- le milieu NiTSCH-NITSCH est celui qui favorise le plus le développement du système racinaire; il se classe en $1^{\text {er }}$ dans les 3 types de milieux pour le rapport racine/tige. Les racines sont bien ramifiées, de grosseur moyenne; les tiges sont normales, les feuilles chlorophylliennes (fig. 2) ;

- le milieu MURASHIGE-SKOOG se classe en $2^{\mathrm{e}}$ position pour le rapport racine/tige dans 2 types de milieux sur 3. Les plantules sont très homogènes, les racines bien développées mais peu ramifiées ;

- le milieu MILLER se classe en $3^{\mathrm{e}}$ position pour le rapport racine/tige dans 2 types de milieux sur 3 . Les plantules sont très hétérogènes en fin d'essai, les tiges sont normales et les racines peu ramifiées;

- le milieu SCHENK-HILdEBRANDT se classe en $4^{\mathrm{e}}$ position pour le rapport racine/tige, les racines sont courtes, épaisses, peu ramifiées, les tiges sont très développées avec des feuilles larges.

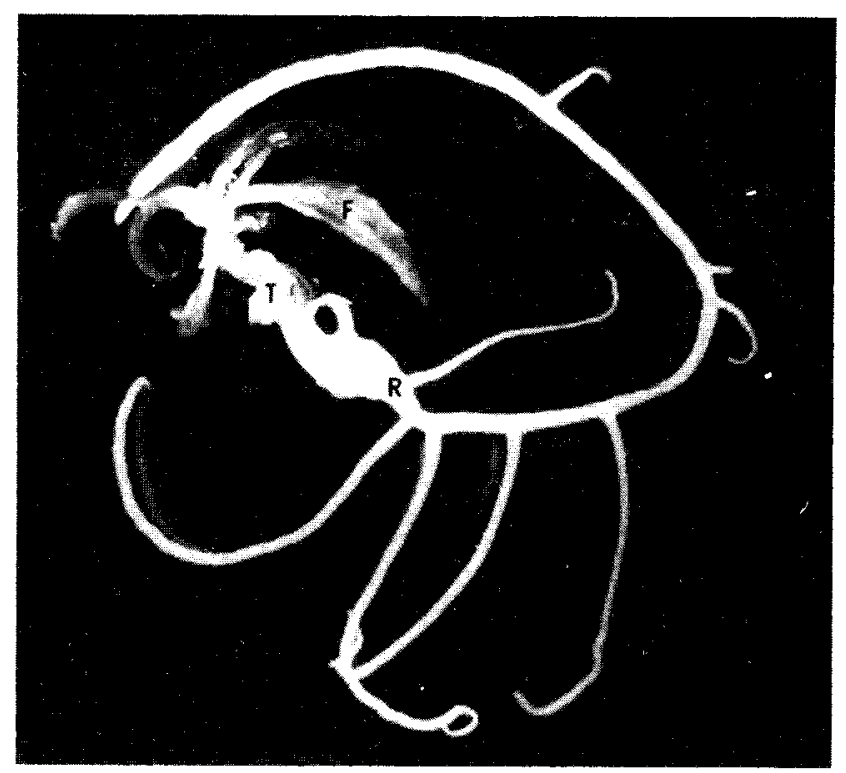

Figure 2

Axe embryonnaire après $19 j$ de croissance dans la solution nutritive de NITSCH-NITSCH $(\times 2)$.

Feuilles $(F)$, tige $(T)$, racines $(R)$.

Embryonic axis after 19 days of growth in the NitsCH-NiTsCH nutrient solution $(\times 2)$.

Leaves $(F)$, stem $(T)$, roots $(R)$.

En définitive, chaque solution minérale utilisée se distingue des autres non seulement par son aptitude à induire la formation d'une plus ou moins grande quantité de matière sèche, mais aussi par son aptitude à favoriser le développement préférentiel soit de la partie racinaire de l'axe soit de sa partie aérienne; enfin elle peut avoir un effet sur la morphologie de la plantule.

\section{CONCLUSION}

En culture d'embryons, le recours aux substances de croissance n'est pas souhaitable car elles entraînent 
le plus souvent une dédifférenciation des tissus. On ne dispose donc plus d'un moyen efficace susceptible d'induire un développement préférentiel de la radicule ou de la gemmule de l'embryon. En dehors des substances de croissance, les autres constituants du milieu (sels minéraux, vitamines, acides aminés, sucres, etc...) ont-ils une activité plus ou moins favorable au développement de la radicule ou de la gemmule ? Il semble que oui, au moins en ce qui concerne les sels minéraux.

Les essais que nous venons de présenter ne sont pas suffisamment précis pour qu'il soit possible de dire quel est l'élément minéral qui agit sur la tige, la racine ou qui provoque des modifications morphologiques; il faudrait pour cela faire varier chaque élément du milieu ou des éléments antagonistes pour percevoir cet effet. Cependant, ils nous ont permis d'éliminer les milieux peu favorables à la synthèse de matière sèche ou les milieux qui favorisent un développement non souhaité d'une partie de l'axe (milieu SCHENKHILDEBRANDT plus favorable à la tige qu'à la racine par exemple).
Nous pensons que le milieu de NitsCH-Nitsch qui favorise beaucoup le développement racinaire devrait donner de bons résultats pour la culture d'embryons immatures du pêcher, car les embryons qui ont un système racinaire bien développé ont généralement un taux de survie élevé. D'une manière générale, il nous semble que les axes embryonnaires du pêcher sont de bons révélateurs des qualités et des défauts des milieux destinés aux cultures d'embryons dans lesquels la présence de substances de croissance est le plus souvent prohibée. Leur utilisation à cet effet offre plusieurs avantages :

- on peut en disposer toute l'année,

- ils contiennent, à l'état d'ébauches, les principaux organes de la plante adulte,

- ils sont faciles à prélever aseptiquement,

- ils réagissent avec sensibilité aux variations des milieux de culture,

- ils donnent des résultats reproductibles.

Reçu le 20 septembre 1983. Accepté le 5 juin 1984.

\section{RÉFÉRENCES BIBLIOGRAPHIQUES}

Davidson O. W., 1963. The germination of «non viable » peach seeds. proc. Am. Soc. Hortic. Sci., 30, 129-132.

Heller R., 1953. Recherches sur la nutrition minérale des tissus végétaux cultivés « in vitro ». Ann. Sci. Nat., Bot. Biol. Vég., II sér., 14, 1-233.

Lammerts W. E., 1942. Embryo culture : an effective technique for shortening the breeding cycle of deciduous trees and increasing the germination of hybrid seed. Am. J. Bot., 29, 166.

Lesley J. W., Bonner J., 1952. The development of normal peach seedling from seeds of early maturing varieties. Proc. Am. Soc. Hortic. Sci., 60, 238-242.

Mauney J. R., 1961. The culture in vitro of immature cotton embryos. Bot Gaz., 122, 205-209.

Miller C. O., 1965. Evidence for the natural occurence of zeatin and derivatives compounds from maize which promote cell division. Proc. Nat. Acad. Sci. U.S.A., 54 (4), 1052-1058.

Monet R., 1968. Méthode permettant l'obtention de plantes viables à partir d'embryons de variétés très précoces chez le pêcher. Ann. Amélior. Plant., 29 (1) 79-88.

Monnier M., 1976. Culture in vitro de l'embryon immature de Capsella bursa-pastoris Moench. Rev. Cytol. Biol. Vég., 39, 1-20.
Murashige T., Skoog F., 1962. A revised medium for rapid growth and bioassays with tobacco tissue cultures. Physiol. Plant., 15, 473-497.

Nitsch J. P., Nitsch P., 1969. Haploid plants from pollen grains. Science, 163, 85-87.

Quoirin M., Lepoivre P., 1977. Etude de milieux adaptés aux cultures « in vitro » de Prunus. Symposium on tissue culture for Horticultural purposes. Belg. Acta Hortic., 78, 437-442.

Schenk R. V., Hildebrandt A. C., 1972. Medium and techniques for induction and growth of monocotyledonous and dicotyledonous plant cell cultures. Can. J. Bot., 50 (1), 199-204.

Theobald W. L., Hough F. L., 1960. The relationship between stage of peach embryo development and seedling growth and survival. Proc. Am. Soc. Hortic. Sci., 75, 163-170.

Toledo W., Hugard J., Jonard R., 1980. Amélioration de la technique d'obtention de plants de semis de pêcher (Prunus persica Batsch) à partir de graines du cultivar de maturité précoce « Springtime ». C.R. Acad. Sci. Paris, 290, D, 539-542.

White P. R., 1936. Plant tissue cultures. Bot. Rev., 2, 419-437. 